\title{
Periodic unmixing of a binary metallic vapor
}

\author{
J. Maul, I. Strachnov, S. Karpuk, T. Schilling, A. Oelsner, P. Bernhard, H. J. Elmers, G. Schönhense, and G. Huber \\ Institut für Physik, Staudingerweg 7, Johannes Gutenberg-Universität, D-55099 Mainz, Germany \\ (Received 12 May 2005; revised manuscript received 26 July 2005; published 25 October 2005)
}

\begin{abstract}
We report on a type of surface structuring after short pulse laser ablation of a binary alloy. We observe the emergence of a concentric ring structure with changing elemental composition. The composition changes are interpreted by condensation of the ambient ablation vapor due to stress wave excitations in the ablation spot.
\end{abstract}

DOI: 10.1103/PhysRevB.72.155431

PACS number(s): 79.20.Ds, 52.38.Mf, 68.03.Fg

\section{INTRODUCTION}

When a metallic surface is irradiated by a short laser pulse, the absorbed photon energy dissipates into thermal energy of the electron gas on a femtosecond time scale. The electronic excitation thermalizes with the ambient lattice of the solid on a picosecond time scale. ${ }^{1,2}$ If the temperature is sufficiently high, melting and vaporization set in, and finally a release of matter proceeds on a nanosecond time scale. ${ }^{3}$ In this context, the laser pulse driven removal of matter is denoted as "laser ablation," which is a widely used mechanism, e.g., in material processing ${ }^{4,5}$ and in the microanalysis of solid samples. ${ }^{6}$

Furthermore, laser ablation offers unique possibilities to investigate condensed matter under extreme conditions. When high photon fluxes are applied to surfaces, metastable phases of matter can emerge on short time scales. ${ }^{7}$ In this transient regime, high pressures in the range of several gigapascal can be expected, from which strong acoustic perturbations ${ }^{8}$ as well as thermoelastic and ablation-induced stress $^{9}$ can emerge.

The usual aspect of a solid surface after pulsed laser irradiation is the formation of an ablation crater, as frequently reported, e.g., in Refs. 10 and 11. The depth of the crater mainly depends on the deposited pulse energy, and the shape of the crater strongly depends on the substrate material as well as on the duration of the laser pulse. ${ }^{12}$ In some cases, morphological features such as columns ${ }^{13}$ and ripples ${ }^{14}$ have been seen. Furthermore, the appearance of planar wavelike patterns was reported after multipulse nanosecond ablation, arising from cumulative growth within an instable surfaceplasma interface. ${ }^{15}$

Here, we have observed permanent concentric ring structures after multiple short pulse (i.e., nanosecond) laser ablation. We suggest that this pattern can be interpreted by transient standing stress waves in the ablation zone. We base this suggestion on the elemental analysis of the concentric ring pattern, which became visible after the ablation vapor had interacted with the underlying surface.

This paper is structured as follows: In Sec. II, the main observations are presented based on a morphological analysis of the ablation spot. From this, an interpretation is given on the basis of thermodynamic vapor phase considerations, which are combined with considerations about stress wave excitations within the ablation spot (Sec. III). Further experimental evidence is presented to corroborate the main hypothesis (Sec. IV). Finally the observed concentric patterns are analyzed with respect to the standing stress wave hypothesis (Sec. V).

\section{MORPHOLOGIC ANALYSIS OF LASER-INDUCED SURFACE STRUCTURES}

In a first experiment we have used a binary alloy (brass CuZn31, 69\% of copper and $31 \%$ of zinc) covered by a high absorption coating of 1-2 $\mu \mathrm{m}$ thickness (consisting of $38 \%$ chemically bound copper, $2 \%$ bound zinc, and a main part of oxygen). This target has been irradiated at normal incidence under atmospheric conditions by a series of 20 laser pulses from the third harmonic of a Nd:Yttrium aluminium garnet (YAG) laser at 355-nm wavelength (Quantel Brilliant, pulse duration $\tau \sim 4 \mathrm{~ns}$, laser fluence $\sim 110 \mathrm{~mJ} / \mathrm{cm}^{2}$; a segment of the measured laser beam profile is given as inset in Fig. 1).

A single laser pulse could not remove all the coating from the ablation spot, and the ablation process was repeated more than ten times. When the high absorption coating has been completely removed (after $\sim 20$ pulses), the alloy surface appears with a ring pattern as shown in Fig. 1 (left).

In the following, we concentrate on purely morphological investigations. First a microscopic image of a smaller region within the ablation spot has been taken by means of a scanning electron microscope (SEM Jeol $6400 \mathrm{~F}$ ), as shown in Fig. 1 (right). The concentric structures show up as statistical arrangements of much smaller microstructures along the ring fronts, typically in the range of $10-40 \mu \mathrm{m}$.

To perform a spatially resolved elemental surface analysis, a scanning energy dispersive $\mathrm{x}$-ray spectrometer (EDX,
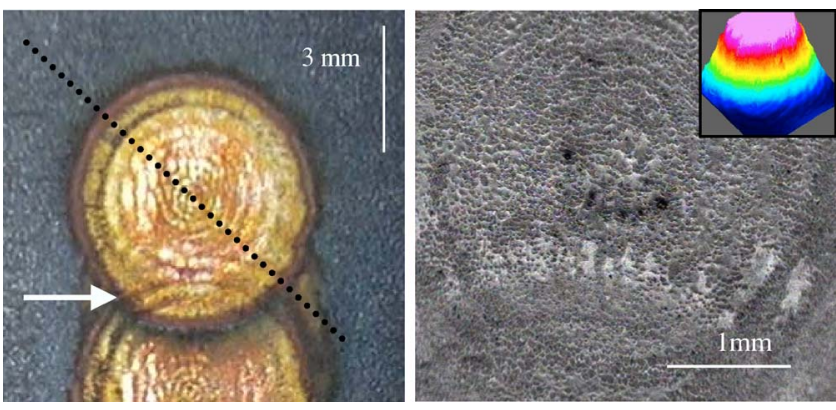

FIG. 1. (Color online) Surface of coated brass after irradiation with a series of 4-ns laser pulses of $110 \mathrm{~mJ} / \mathrm{cm}^{2}$. The dotted line is used for further analysis. Left: photographic image; right: SEM micrograph. The inset shows the measured laser beam profile. 


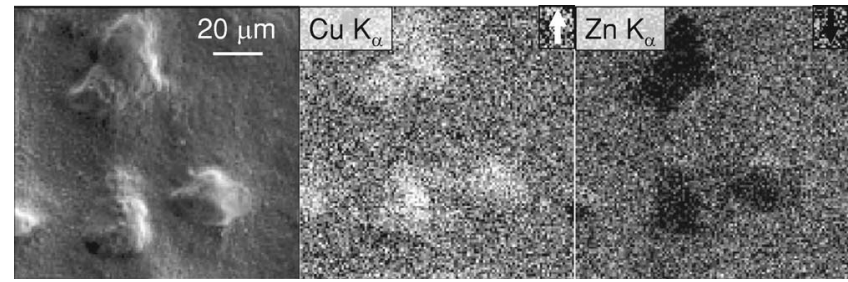

FIG. 2. SEM micrograph (left) and elemental scans of copper (middle) and zinc (right) on the surface. Increasing copper content and, respectively, decreasing zinc content, is observed on the microstructure surface.

Spectrometer Jeol JXA 8900 RL; energy resolution $\Delta E \sim 0.13 \mathrm{keV}$ ) has been used for an energy dispersive observation of the characteristic $K \alpha$ x-ray emissions ( $\mathrm{Cu} \mathrm{K} \alpha: 8.04 \mathrm{keV}$; Zn K $\alpha: 8.63 \mathrm{keV})$. The spatial resolution of $<2 \mu \mathrm{m}$ has been sufficient to resolve local changes in the elemental composition of the sample surface. In Fig. 2 (left), a domain of $\sim 120 \mu \mathrm{m} \times 120 \mu \mathrm{m}$ in the middle of a concentric ring front is mapped by a SEM micrograph. This domain includes three of such microstructures with a size of $\sim 20 \mu \mathrm{m}$. The same domain has been subjected to a spatially resolved elemental EDX scan. The scanning results are presented for copper in Fig. 2 (middle), and for zinc in Fig. 2 (right). From these gray value representations, an increase of the average copper concentration of $\sim+(11 \pm 2) \%$, and vice versa, an average decrease of the zinc concentration of $\sim-(11 \pm 2) \%$ is registered. These inspections unambiguously show small cupreous protuberances on a clear brass ground.

To recap, the laser-irradiated coated brass surface exhibits concentric rings constisting of microsctructures of increased copper contents. In the following, an explanation is given that consolidates considerations about laser-induced stress with thermodynamical arguments.

\section{INTERPRETATION AND DISCUSSION}

From the above observations, we propose an explanation for the laser-induced emergence of cupreous microstructures within the concentric rings. For this purpose we combine considerations about the photomechanical response of the irradiated surface with a thermodynamic ablation vapor analysis.

The excitation of stress waves during nanosecond laser ablation is a well-documented phenomenon. When a nanosecond laser pulse strikes a surface and removes a significant amount of material, the recoil momentum of the ablation front induces photomechanical stress propagating through the material. ${ }^{16,17}$ Thereby a part of the stress wave travels across the surface boundary into the surrounding atmosphere. ${ }^{18}$ Here we consider the possibility that another part of the wave is partially reflected at the sharp boundaries of the laser-induced melting pot. These boundaries are due to abrupt changes in density from molten to solid material. ${ }^{7}$

Recently, photomechanical perturbations have been investigated in great detail for nanosecond ablation. ${ }^{9}$ It was shown that the laser-induced stress can be split into at least two

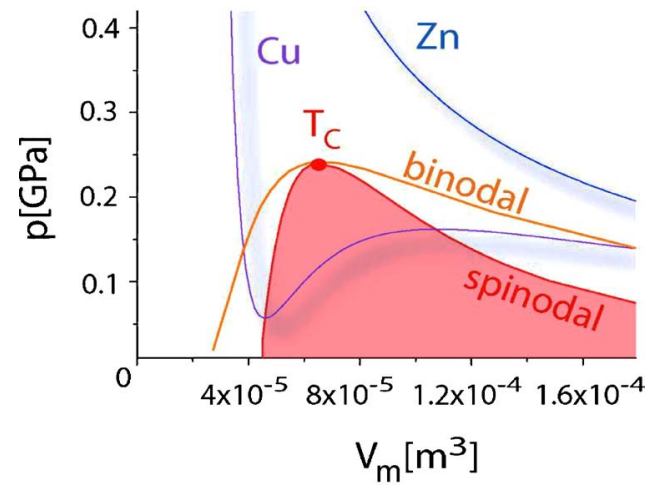

FIG. 3. (Color online) Van der Waals diagram of a copper vapor and a zinc vapor at a temperature of $4500 \mathrm{~K}$. $V_{m}$ denotes the corresponding molar volumina-the spinodal and binodal line are plotted for the copper phase.

components, both unipolar (transversal) ablation-induced stress from the recoil momentum of the removed material, and a (transversal) thermoelastic excitation identified by a compression or tension wave that is bipolar with respect to the unperturbed flat surface.

For a better understanding of the ring composition, it is interesting to investigate the vapor phase thermodynamics. Therefore, the van der Waals diagram for both copper and zinc was calculated for an exemplary temperature of $T=4500 \mathrm{~K}$, which is in agreement with the energetic balance of the laser irradiation condition (Fig. 3). The critical temperature of the vapor-liquid transition in the zinc phase is $T_{C}^{\mathrm{Zn}}=3380 \mathrm{~K}$, while that of the copper phase is $T_{C}^{\mathrm{Cu}}=5148 \mathrm{~K}$. Therefore, at $T=4500 \mathrm{~K}$ the zinc phase has already entered an almost ideal gas regime, with a relatively high partial pressure compared to the coexisting copper vapor phase. The zinc vapor is therefore rather robust against compression and thermal fluctuations, whereas the copper vapor phase tends to enter a regime of thermodynamic metastability, marked by the binodal line. ${ }^{19,20}$ The more refractory copper (boiling temperatures $T_{b}^{\mathrm{Cu}}=2839 \mathrm{~K}, T_{b}^{\mathrm{Zn}}=1148 \mathrm{~K}$ at atmospheric pressure) thereby enters a supercooled phase close to the metal surface and tends to recondensate on small perturbations.

With respect to the thermodynamic vapor phase considerations, the appearing structure can be interpreted in terms of stress wave excitations within the melting zone of the ablation spot: In this picture, the structured cupreous condensation of the ablation vapor is caused by coupling of the surface excitation to the vapor phase in the immediate vicinity of the surface, favoring the condensation of the copper along fronts of increased pressure. Following the argumentation by Refs. 21 and 22, especially bipolar stress waves propagating along the surface can promote rapid nucleation over a large spatial scale. In combination with a standing wave mode formation within the transient melting volume, the concentric microstructure patterns are interpreted by stress-induced nucleation of the less volatile copper constituent in the metastable binary vapor phase. The above considerations hold for a wide temperature range of $\Delta T \sim 1770 \mathrm{~K}$ between the critical temperatures of both constituents copper and zinc. 

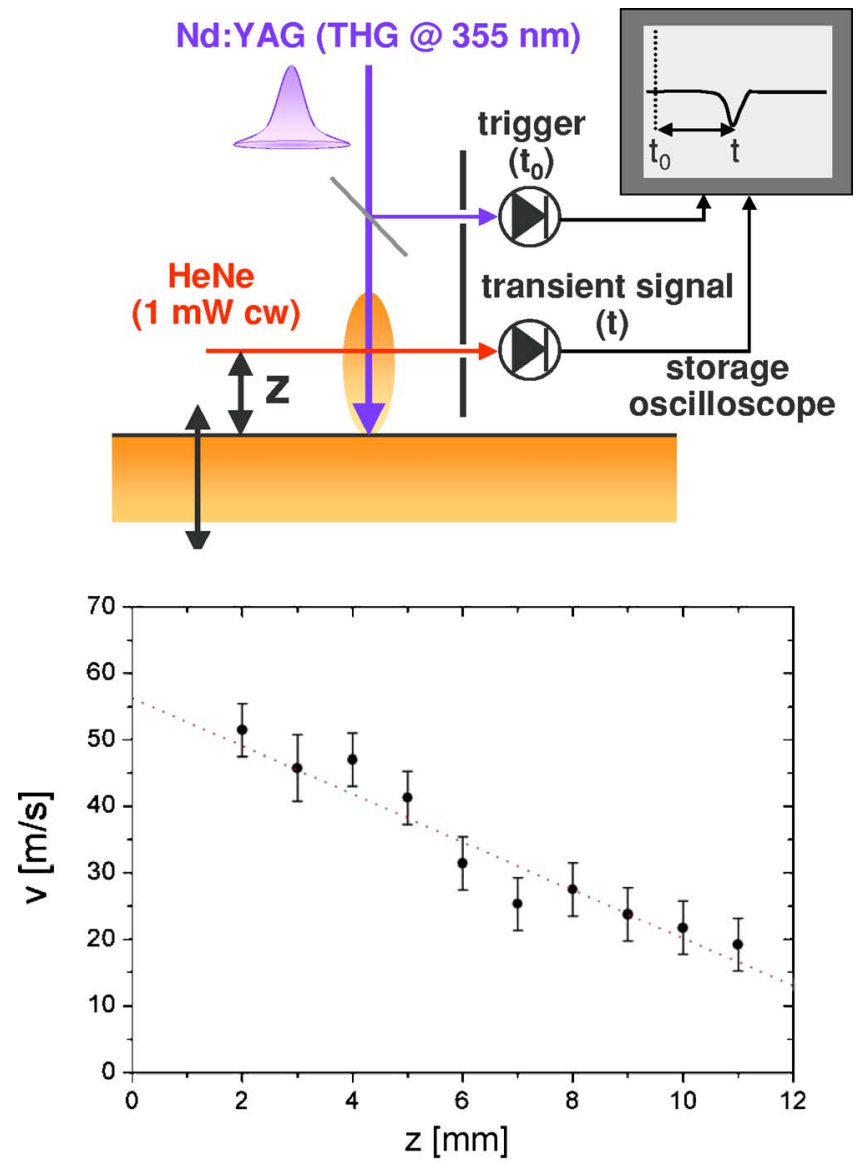

FIG. 4. (Color online) Top: Schematic view of the pump-probe experiment for the determination of the ablation front velocity of the absorption coating layer. Bottom: Spatial change of the ablation front velocity, showing significant deceleration at atmospheric conditions. An expansion velocity of $\sim(56 \pm 5) \mathrm{m} / \mathrm{s}$ is extrapolated close to the surface.

\section{SUPPLEMENTARY EXPERIMENTAL EVIDENCE}

In order to demonstrate the confining influence of the ablation front on the underlying vapor, we have further determined the expansion velocity of the ablation front under atmospheric conditions.

The schematic setup is presented in Fig. 4 (top). In brief, the ablation front starting from a movable target partially scatters light from a traversing continuous wave HeNe laser beam $(\sim 1 \mathrm{~mW}, \lambda=633 \mathrm{~nm})$, which is coupled into a fast photodiode. From the distance $z$ between the target and the HeNe laser beam, and the time $t$ between the ablation laser pulse and the transient signal from the ablation front, the local expansion velocity $v:=z / t$ is determined (Fig. 4, bottom).

Using this setup, we have determined the expansion velocity of the high absorption front in the vicinity of the ablation target surface to $\sim(56 \pm 5) \mathrm{m} / \mathrm{s}$, which is more than one order of magnitude below the expansion velocity of ablation fronts typically obtained under vacuum conditions. ${ }^{16,17}$ The reduced front velocity augments the time scale for a vapor-surface interaction, and therefore causes a stronger vapor confinement near the surface.
The ablation experiment has been further performed under different laser irradiation conditions. At the maximum fluence of $\sim 220 \mathrm{~mJ} / \mathrm{cm}^{2}$ of the unfocused $\mathrm{Nd}$ : YAG laser, cupreous surface structures were still observed. After a strong increase of the fluence by focusing the laser beam to an ablation spot size of $\sim 1 \mathrm{~mm}$ in diameter however, no more structuring was observed and a cupreous ablation residue consisting of the bulk brass composition was obtained.

The fact that the ring structures were still observed at the maximum laser fluence of $220 \mathrm{~mJ} / \mathrm{cm}^{2}$, which should in a simplified picture exceed the critical point of both constituents, might be attributed to an increasing laser light shielding of the forming ablation plume with increasing laser fluence, ${ }^{23}$ in particular due to increasing photoionization. ${ }^{24}$ In the focused laser spot at maximum laser pulse energy, however, the critical point of both constituents copper and zinc is trespassed, as is recognized from the emergence of the pure brass surface.

In order to rule out possible condensation of copper-rich particles from the layer itself, supplementary experiments have been performed on uncoated brass as well as on brass coated with a carbon-based layer that is free of zinc and copper. The cupreous structuring has been observed in both cases under air condition; however, more clearly in the presence of the coated target.

For a better understanding of the influence of the surrounding atmosphere, these tests have also been carried out under argon gas pressure as well as under vacuum conditions $\left(\sim 10^{-2}\right.$ mbar $)$. Thereby it turned out that the specific kind of atmosphere hardly influences the experiment, but that no clear structuring became visible from the uncoated target under vacuum condition. This again demonstrates the role of the coating layer, which is in general not necessary for the emergence of those structures, but shows some assisting influence. During the ablation process the high-absorption coating possibly confines the underlying vapor by transferring its momentum to the surface.

Furthermore, the two partly overlapping structures shown in Fig. 1 have been produced by two successive ablation cycles with partly overlapping areas. It is interesting to note that the ring structure from the previously irradiated spot is not destroyed by the spot that has been irradiated at a later time, which is seen from the morphologic analysis of the domain marked by an arrow in Fig. 1. Here the upper spot has been irradiated after the lower spot has been subjected to ablation. This observation, together with the observation that the ring pattern is even emphasized with increasing shot number, indicates substrate interactions of the pattern with the ablation vapor, and excludes smoothing by melting during the laser irradiation process. Moreover, the pattern formation cannot be related to distillation during the evaporation process, which should result in porelike depressions instead of raised microparticles onto a flat surface.

\section{STRUCTURAL ANALYSIS}

In the laser-induced stress wave picture, the front distances are interpreted as effective wavelengths of the wavelike surface perturbation. The radial change of the front dis- 


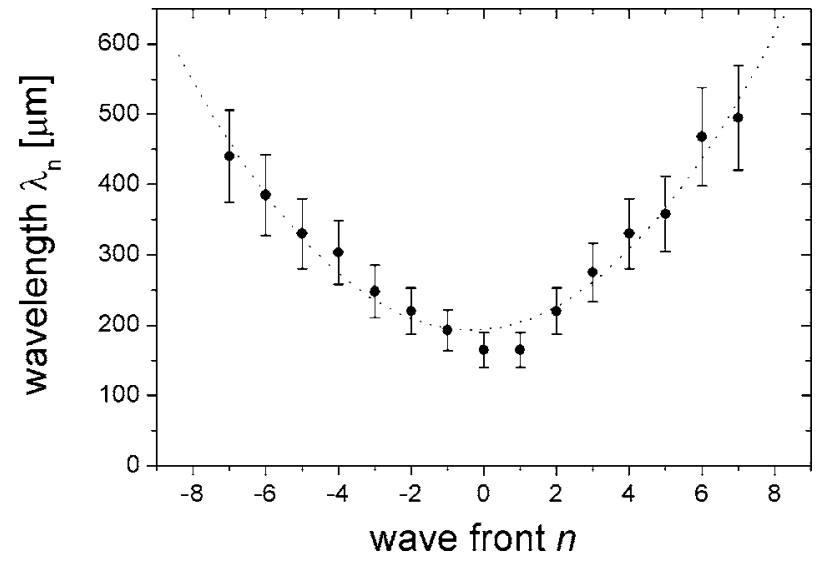

FIG. 5. Spatial dependence of the wavelength, as extracted along the dotted line in Fig. 1. The wave front distribution is interpreted by means of local changes of the thermodynamic properties within the ablation zone, as it is explained more detailed in the text.

tances is therefore interpreted as the dispersion of the stress wave mode building up in the ablation zone. The dispersion of the wave itself directly depends on the density profile of the transient melting spot in the ablation zone, and the density profile is governed by the corresponding temperature profile. In Fig. 5, the radial wavelength dependency is shown, indicating a strong increase to the margin of the ablation zone. A polynomial function has been used as a guideline to emphasize the increase of the wavelengths from the center to the margin. This can be understood by the fact that the peak intensity of the laser pulses induces the highest pressure, respectively, the highest density in the ablation spot, thus giving rise to the shortest wavelength $\lambda(T)=c(T) \nu$ where $\nu$ is the frequency of the mode, and the speed of sound is given by $c(T)=\sqrt{1 / \kappa(T) \rho(T)}$ with the compressibility $\kappa$ and the density $\rho$ of the fluid. It is now possible to extract $\kappa \cdot \rho$ from this, but it is not possible here to separate their influence on $c(T)$. In some temperature regimesmainly near the critical point $-\kappa$ is expected to change strongly, in others, however, $\rho$. Thus, the wavelength dispersion can be seen as a direct expression of the combined quantity $\kappa(T) \rho(T)$.

An alternative explanation for the formation of a standing wave might be provided by the slowly expanding overlay during the ablation process. Given a dense expanding coating layer that confines the ablation vapor on a rather long (i.e., microsecond) time scale, a transient wave excitation might be reflected and result in a transient standing wave.

We have used the simplified picture of a van der Waals gas for the condensation in a nonequilibrium phase driven by a transient pressure wave. In other experiments, droplet ejection and droplet formation have also been observed in the early stages of the ablation process (e.g., in Ref. 25). These droplets can directly serve as condensation nuclei for the ambient binary vapor, thus resulting in a similar condensation pattern.

\section{CONCLUSION}

In conclusion, we have observed a type of surface structuring after the nanosecond laser ablation of a binary alloy. Concentric ring alignments of microstructures with changing elemental composition have been identified. We suggest that this pattern indicates stress wave excitations within the ablation zone by recondensation of metastable ablation vapor along certain pressure fronts.

\section{ACKNOWLEDGMENTS}

The authors would like to thank D. von der Linde for helpful discussion, as well as B. Schulz-Dobrick and S. Fölling for experimental support. T.S. acknowledges support by the DFG Emmy Noether Programm.
${ }^{1}$ C. Momma, B. N. Chichkov, S. Nolte, A. v. Alvensleben, A. Tünnermann, H. Welling, and B. Wellegehausen, Opt. Commun. 129, 134 (1996).

${ }^{2}$ K. Sokolowski-Tinten, J. Bialkowski, and D. von der Linde, Phys. Rev. B 51, 14186 (1995).

${ }^{3}$ D. von der Linde and K. Sokolowski-Tinten, Appl. Surf. Sci. 154, 1 (2000).

${ }^{4}$ D. Bäuerle, Laser Processing and Chemistry, 3rd ed. (Springer, Berlin, 2000).

${ }^{5}$ W. M. Steen, J. Opt. A, Pure Appl. Opt. 5, 3 (2003).

${ }^{6}$ A. Audétat, D. Günther, and C. A. Heinrich, Science 279, 2091 (1998).

${ }^{7}$ K. Sokolowski-Tinten, J. Bialkowski, A. Cavalleri, D. von der Linde, A. Oparin, J. Meyer-ter-Vehn, and S. I. Anisimov, Phys. Rev. Lett. 81, 224 (1998).

${ }^{8}$ K. Sokolowski-Tinten, C. Blome, C. Dietrich, A. Tarasevitch, M. Horn von Hoegen, D. von der Linde, A. Cavalleri, J. Squier, and M. Kammler, Phys. Rev. Lett. 87, 225701 (2001).

${ }^{9}$ A. Rohlfing, C. Menzel, L. M. Kukreja, F. Hillenkamp, and K.
Dreisewerd, J. Phys. Chem. B 107, 12275 (2003).

${ }^{10}$ R. Stoian, D. Ashkenasi, A. Rosenfeld, and E. E. B. Campbell, Phys. Rev. B 62, 13167 (2000).

${ }^{11}$ J. Ihlemann, B. Wolff, and P. Simon, Appl. Phys. A: Solids Surf. 54, 363 (1992).

${ }^{12}$ X. Zeng, X. L. Mao, R. Greif, and R. E. Russo, Appl. Phys. A: Mater. Sci. Process. 80, 237 (2005).

${ }^{13}$ T.- H. Her, R. J. Finlay, C. Wu, S. Deliwata, and E. Mazur, Appl. Phys. Lett. 73, 1673 (1998).

${ }^{14}$ J. Bonse, S. Baudach, J. Krüger, W. Kautek, and M. Lenzer, Appl. Phys. A: Mater. Sci. Process. 74, 19 (2002).

${ }^{15}$ L. K. Ang, Y. Y. Lau, R. M. Gilgenbach, H. L. Spindler, J. S. Lash, and S. D. Kovaleski, J. Appl. Phys. 83, 4466 (1998).

${ }^{16}$ J. Lee, M. F. Becker, and J. W. Kreto, J. Appl. Phys. 89, 8146 (2001).

${ }^{17}$ J. Maul, S. Karpuk, and G. Huber, Phys. Rev. B 71, 045428 (2005).

${ }^{18}$ X. Zeng, X. Mao, S. -B. Wen, R. Greif, and R. E. Russo, J. Phys. D 37, 1132 (2004). 
${ }^{19}$ D. Jou, J. Casas-Vazquez, and G. Lebon, Extended Irreversible Thermodynamics, 3rd ed. (Springer, Berlin, 2001).

${ }^{20}$ J. L. Sengers, How Fluids Unmix (KNAW, Amsterdam, 2002).

${ }^{21} \mathrm{~S}$. aus der Wiesche, C. Rember, and E. P. Hofer, Heat Mass Transfer 35, 143 (1999).

${ }^{22}$ A. Vogel and V. Venugopalan, Chem. Rev. (Washington, D.C.)
103, 577 (2003)

${ }^{23}$ R. E. Russo, X. Mao, and S. S. Mao, Anal. Chem. 74, 71A (2002).

${ }^{24}$ J. J. Chang and B. Warner, Appl. Phys. Lett. 69, 473 (1996).

${ }^{25}$ C. Körner, R. Mayerhofer, M. Hartmann, and H. W. Bergmann, Appl. Phys. A: Mater. Sci. Process. 63, 123 (1996). 